\title{
Failure with acquired resistance of an optimised bedaquiline-based treatment regimen for pulmonary Mycobacterium avium complex disease
}

\begin{abstract}
To the Editor:
5 years ago, a then 50-year-old woman presented with long-standing fatigue, dyspnoea and a chronic productive cough. Based on a computed tomography scan of the thorax and multiple positive cultures, she was diagnosed with nodular-bronchiectatic Mycobacterium avium pulmonary disease; she was also found to have a heterozygous F508del CFTR gene mutation. She commenced therapy with rifabutin $300 \mathrm{mg}$ once daily, ethambutol $1200 \mathrm{mg}$ once daily and azithromycin $500 \mathrm{mg}$ once daily. After 15 months of ongoing symptoms, radiographic deterioration and persistent culture positivity, clofazimine $100 \mathrm{mg}$ once daily and thrice weekly intravenous amikacin $15 \mathrm{mg} \cdot \mathrm{kg}^{-1}$ were added to the regimen. Amikacin was halted after 4 months; the remaining four drugs were continued. The dose of azithromycin was lowered to $250 \mathrm{mg}$ once daily after 9 months because of hearing loss.
\end{abstract}

After 2 years of stable symptoms and radiology but persistent culture positivity, rifabutin was replaced by bedaquiline, in the $400 \mathrm{mg}$ once daily for 2 weeks and then $200 \mathrm{mg}$ thrice weekly dosing regimen used for tuberculosis. Azithromycin was switched to clarithromycin $500 \mathrm{mg}$ twice daily, and ethambutol and clofazimine were maintained. Therapeutic drug monitoring on the last day of the daily bedaquiline dosing (i.e. at the end of the loading phase, thus at steady state) showed peak and trough bedaquiline plasma concentrations of $3.4 \mathrm{mg} \cdot \mathrm{L}^{-1}$ and $1.2 \mathrm{mg} \cdot \mathrm{L}^{-1}$, respectively, and a total exposure (area under the curve (AUC) over $24 \mathrm{~h}$ ) of $\sim 43 \mathrm{~h} \cdot \mathrm{mg} \cdot \mathrm{L}^{-1}$. The ethambutol peak serum concentration was $3.0 \mathrm{mg} \cdot \mathrm{L}^{-1}$ with an AUC of $28.5 \mathrm{~h} \cdot \mathrm{mg} \cdot \mathrm{L}^{-1}$ and the clarithromycin peak plasma concentration was $1.4 \mathrm{mg} \cdot \mathrm{L}^{-1}$.

This bedaquiline-containing regimen, too, did not lead to culture conversion. Moreover, the M. avium isolates obtained during and after bedaquiline treatment showed an increase in bedaquiline and clofazimine minimum inhibitory concentration (MIC), and by whole-genome sequencing, a new mutation in the regulator of the efflux pump involved in bedaquiline and clofazimine resistance (figure 1).

After 12 months and with persistent symptoms and culture positivity, bedaquiline was stopped and a bilobectomy of the right upper and middle lobe was performed. Post-operatively, rifabutin $300 \mathrm{mg}$ once daily and amikacin liposomal inhalation solution were added to the clofazimine-ethambutolclarithromycin regimen. Although surgical samples were culture positive, sputum culture conversion was attained after surgery and had lasted for 6 months at the time of writing.

Throughout the course of treatment, all M. avium isolates remained macrolide- and amikacin-susceptible and no QTc interval prolongation was observed by ECG monitoring.

Bedaquiline was developed for treatment of multidrug-resistant tuberculosis (MDR-TB) but has also been explored for use in disease caused by nontuberculous mycobacteria [1]. To date, bedaquiline treatment and outcome data of 13 patients with M. avium complex pulmonary disease (MAC-PD) have been reported and these were not favourable [1]. After an initial clinical, radiological and microbiological response [1], most patients' treatment failed with the emergence of mutational resistance to bedaquiline [2]. Most of

@ERSpublications

In its current dose, bedaquiline is probably not a viable treatment option for treatment-refractory MAC-PD; even with adequate exposure, treatment fails due to acquired resistance documented by increasing MICs and whole-genome sequencing http://bit.ly/2YvaTMM

Cite this article as: Zweijpfenning SMH, Schildkraut JA, Coolen JPM, et al. Failure with acquired resistance of an optimised bedaquiline-based treatment regimen for pulmonary Mycobacterium avium complex disease. Eur Respir J 2019; 54: 1900118 [https://doi.org/10.1183/13993003.00118-2019]. 


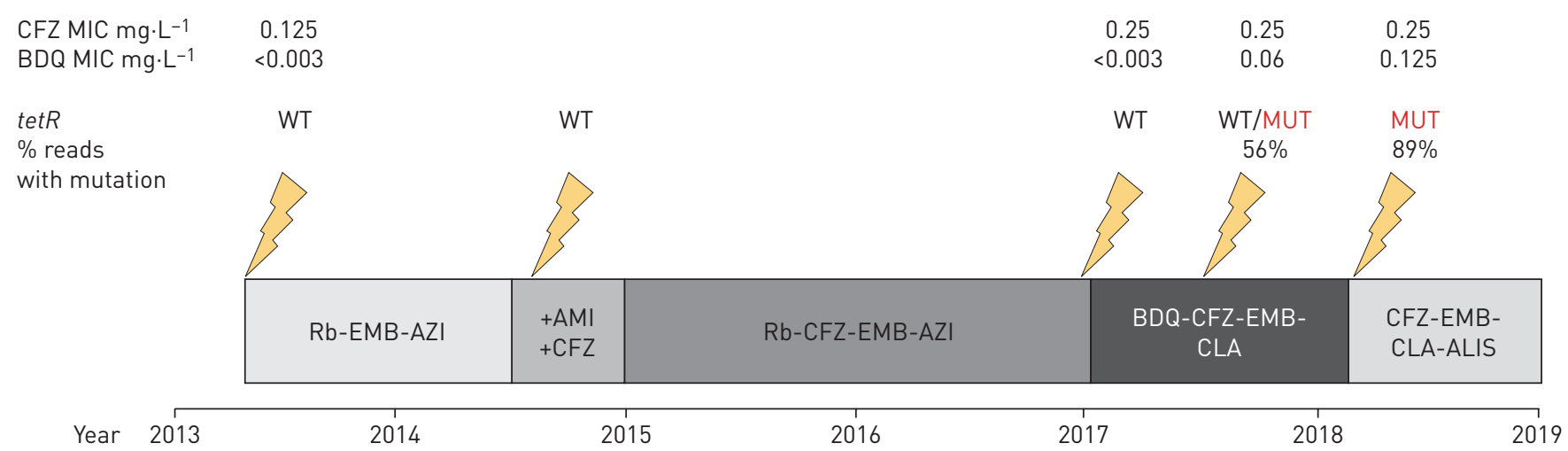

FIGURE 1 Timeline. CFZ: clofazimine; MIC: minimum inhibitory concentration; BDQ: bedaquiline; WT: wild type; MUT: mutant; Rb: rifabutin; EMB: ethambutol; AZI: azithromycin; AMI: amikacin; CLA: clarithromycin; ALIS: amikacin liposome inhalation solution.

these patients used a rifamycin (rifampicin or rifabutin) and bedaquiline as parts of their treatment regimen, and rifampicin is known to decrease the systemic exposure to bedaquiline by $75 \%$ through induction of cytochrome P450 (CYP)3A4 [3]; thus, the emergence of bedaquiline resistance may have resulted from suboptimal exposure.

Clofazimine has been shown to be as active as rifampicin in three-drug regimens that also contain ethambutol and a macrolide [4]; it does not affect the pharmacokinetics of bedaquiline [5] and is synergistic with bedaquiline against $M$. avium complex bacteria [6].

Hence, upon introducing bedaquiline, we aimed to optimise its efficacy in the regimen by stopping rifabutin and continuing clofazimine. Furthermore, the change of azithromycin to clarithromycin (an inhibitor of CYP3A4) might cause increased exposure to bedaquiline [7]. The bedaquiline serum concentrations at the end of week 2 (peak $3.4 \mathrm{mg} \cdot \mathrm{L}^{-1}$ and trough $1.2 \mathrm{mg} \cdot \mathrm{L}^{-1}$ ) were at least similar to the peak $\left(2.76 \mathrm{mg} \cdot \mathrm{L}^{-1}\right)$ and trough concentrations $\left(0.73 \mathrm{mg} \cdot \mathrm{L}^{-1}\right)$ reported by vaN HEESwIJK et al. [7], showing that drug-drug interactions were successfully avoided. This is important, as the bacteriostatic effect of bedaquiline against $M$. avium is known to be exposure dependent [6]. Nonetheless, bedaquilineclofazimine-ethambutol-clarithromycin failed to achieve culture conversion. Exposure to ethambutol was adequate but exposure to clarithromycin, as reflected in its peak concentration of $1.4 \mathrm{mg} \cdot \mathrm{L}^{-1}$, was lower than the mean of $3.91 \mathrm{mg} \cdot \mathrm{L}^{-1}$ in MAC-PD patients treated without rifamycins [8]. The low clarithromycin exposure may have contributed to the poor response to therapy. The fact that bedaquiline was a single drug added to an already failing regimen may also have contributed to the poor outcome of the bedaquiline-containing regimen.

Follow-up cultures during bedaquiline therapy revealed an increase in bedaquiline and clofazimine MIC of the causative $M$. avium strain (figure 1), determined by broth microdilution following Clinical and Laboratory Standards Institute guidelines [6]. This finding prompted us to perform whole-genome sequencing of isolates obtained over the entire course of the patient's disease (figure 1). Whole-genome sequencing revealed a $173 \mathrm{~W}>173 \mathrm{R} \mid 2544950 \mathrm{~A}>\mathrm{G}$ mutation in the MAV_2512 locus (reference genome M. avium 104; GenBank accession number CP000479). No mutations were associated with macrolide or aminoglycoside resistance and it showed that all isolates were fewer than six single-nucleotide polymorphisms different, suggesting a persistent monoclonal infection; the MAV_2512 173W>173R $2544950 \mathrm{~A}>\mathrm{G}$ mutation was already present in $56 \%$ of the sequence reads obtained from the $M$. avium isolates after 7 months but only passed the $>80 \%$ threshold in the isolate obtained after 12 months of bedaquiline therapy (figure 1 ). Of note, this mutation only emerged after bedaquiline was added to clofazimine in the regimen. The affected gene encodes the tetR family transcriptional regulator of the MmpL5/MmpS5 drug transporter, which has been shown to be involved in acquired low-level bedaquiline resistance in Mycobacterium intracellulare as well [2]. Its functional counterpart in Mycobacterium tuberculosis is Rv0678, a marR family transcriptional regulator; mutations in this gene also lead to low-level bedaquiline and clofazimine resistance in M. tuberculosis through increased MmpS5/MmpL5 expression [9].

The acquired mutation in the tet $R$ family transcriptional regulator of the MmpL5/MmpS5 drug transporter is likely the mechanism responsible for the increase in the bedaquiline and clofazimine MIC of the causative M. avium strain, and is apparently not prevented by bedaquiline exposures considered adequate in MDR-TB treatment. The current bedaquiline dose was the highest dose in the only published dose-finding study [10], yet the exposures reached with this dose seem too low to exert a microbiological 
effect and do not prevent acquired drug resistance in MAC-PD. Increased doses may be required for a therapeutic effect but their safety remains to be established.

In summary, the patient presented here experienced failure, with acquired bedaquiline resistance, of an optimised bedaquiline-based treatment regimen designed to minimise drug-drug interactions, maximise bedaquiline exposure and exploit bedaquiline-clofazimine synergy. In its current dose, bedaquiline is probably not a viable treatment option for treatment-refractory MAC-PD.

Sanne M.H. Zweijpfenning ${ }^{1}$, Jodie A. Schildkraut ${ }^{2}$, Jordy P.M. Coolen ${ }^{2}$, Carolien Ruesen ${ }^{2}$, Ellen Koenraad ${ }^{2}$, Anne Janssen $^{2}$, Mike M. Ruth ${ }^{2}$, Arjan S. de Jong ${ }^{2}$, Saskia Kuipers ${ }^{2}$, Rob E. Aarnoutse ${ }^{3}$, Cecile Magis-Escurra ${ }^{1}$, Wouter Hoefsloot $\oplus^{1}$ and Jakko van Ingen $\oplus^{2}$

${ }^{1}$ Radboudumc Center for Infectious Diseases, Dept of Pulmonary Diseases, Radboud University Medical Center, Nijmegen, the Netherlands. ${ }^{2}$ Radboudumc Center for Infectious Diseases, Dept of Medical Microbiology, Radboud University Medical Center, Nijmegen, the Netherlands. ${ }^{3}$ Radboudumc Center for Infectious Diseases, Dept of Pharmacy, Radboud University Medical Center, Nijmegen, the Netherlands.

Correspondence: Jakko van Ingen, Dept of Medical Microbiology, Radboud University Medical Center, PO Box 9101, 6500 HB Nijmegen, the Netherlands. E-mail: jakko.vaningen@radboudumc.nl

Received: Jan 162019 | Accepted after revision: March 242019

Conflict of interest: S.M.H. Zweijpfenning has nothing to disclose. J.A. Schildkraut has nothing to disclose. J.P.M. Coolen has nothing to disclose. C. Ruesen has nothing to disclose. E. Koenraad has nothing to disclose. A. Janssen has nothing to disclose. M.M. Ruth has nothing to disclose. A.S. de Jong has nothing to disclose. S. Kuipers has nothing to disclose. R.E. Aarnoutse has nothing to disclose. C. Magis-Escurra has nothing to disclose. W. Hoefsloot has nothing to disclose. J. van Ingen reports grants from Netherlands Organization for Scientific Research (NWO/ZonMW grant Veni 016.176.024), during the conduct of the study.

Support statement: J. van Ingen is supported by a personal grant from the Netherlands Organization for Scientific Research (NWO/ZonMW grant Veni 016.176.024). Funding information for this article has been deposited with the Crossref Funder Registry.

\section{References}

1 Philley JV, Wallace RJ Jr, Benwill JL, et al. Preliminary results of bedaquiline as salvage therapy for patients with nontuberculous mycobacterial lung disease. Chest 2015; 148: 499-506.

2 Alexander DC, Vasireddy R, Vasireddy S, et al. Emergence of $m m p T 5$ variants during bedaquiline treatment of Mycobacterium intracellulare lung disease. J Clin Microbiol 2017; 55: 574-584.

3 Svensson EM, Murray S, Karlsson MO, et al. Rifampicin and rifapentine significantly reduce concentrations of bedaquiline, a new anti-TB drug. J Antimicrob Chemother 2015; 70: 1106-1114.

4 Jarand J, Davis JP, Cowie RL, et al. Long-term follow-up of Mycobacterium avium complex lung disease in patients treated with regimens including clofazimine and/or rifampin. Chest 2016; 149: 1285-1293.

5 Maartens G, Brill MJE, Pandie M, et al. Pharmacokinetic interaction between bedaquiline and clofazimine in patients with drug-resistant tuberculosis. Int J Tuberc Lung Dis 2018; 22: 26-29.

6 Ruth MM, Sangen JJN, Remmers K, et al. A Bedaquiline-clofazimine combination regimen might add activity to the treatment of clinically relevant non-tuberculous mycobacteria. J Antimicrob Chemother 2019; 74: 935-943.

7 van Heeswijk RP, Dannemann B, Hoetelmans RM. Bedaquiline: a review of human pharmacokinetics and drug-drug interactions. J Antimicrob Chemother 2014; 69: 2310-2318.

8 van Ingen $\mathrm{J}$, Egelund $\mathrm{EF}$, Levin $\mathrm{A}$, et al. The pharmacokinetics and pharmacodynamics of pulmonary Mycobacterium avium complex disease treatment. Am J Respir Crit Care Med 2012; 186: 559-565.

9 Andries K, Villellas C, Coeck N, et al. Acquired resistance of Mycobacterium tuberculosis to bedaquiline. PLoS One 2014; 9: e102135.

10 Rustomjee R, Diacon AH, Allen J, et al. Early bactericidal activity and pharmacokinetics of the diarylquinoline TMC207 in treatment of pulmonary tuberculosis. Antimicrob Agents Chemother 2008; 52: 2831-2835. 